\title{
Interdisciplinary Research and Pandemics
}

\section{Reflections from the University of Leicester Pandemics Advanced Study Group, July 2020}

Clare Anderson, Michael Barer, Gavin Brown, Timothy Coats, Sarah Davies, William Green, Anna Hansell, Maggy Heintz, Jason Hughes, Genovefa Kefalidou, Charlotte King, Julie Morrissey, Susan Page, Manish Pareek, Harjinder Sembhi, Joanna Story, Caroline Upton, Louise Wain, Mark Williams. Reflections invited from ASG Critical Friend, Dr Laura Meagher.

\section{Foreword (Professor Lisa Smith, Director of the Leicester Institute for Advanced Studies)}

The Leicester Institute for Advanced Studies (LIAS) was established in 2017 to provide a stimulating and collaborative environment for interdisciplinary research at the University of Leicester, and beyond. The programme of activity in LIAS has been designed to encourage and support researchers from across all academic disciplines to explore new ways of working together, and we have celebrated some amazing successes with interdisciplinary teams over the past three years. With the LIAS vision and values firmly embedded in the university's research culture, we are always looking for new ways to support interdisciplinary excellence - and 2020 presented a new challenge and opportunity for LIAS in the form of the global COVID-19 pandemic.

The COVID-19 crisis is a global challenge that highlights why interdisciplinary research is so important, and it provides LIAS with an exciting opportunity to launch our first thematic programme of activity. The aim of this approach is to catalyse and galvanise an ambitious, challenge-led interdisciplinary research programme focused on pandemics (including, but not exclusive to, COVID19). LIAS' goal is to provide a platform for colleagues from all three faculties to build a thematic research community who will, together, develop and deliver transformative research.

Our first step was to convene an 'Advanced Study Group' (ASG). An ASG comprises research leaders across a range of disciplines, and provides an opportunity to work on ideas or interlinked research problems in order to set the landscape, priorities and potential sub-themes for the future programme. The ASG is intended to provide its members with the opportunity to think in new interdisciplinary ways, discuss and test ideas, and align the pandemics theme with University of Leicester research strengths. In order to support this goal, and provide independent and external feedback, we invited Dr Laura Meagher (Edinburgh) to be the ASG 'Critical Friend' and facilitator.

This Working Paper is the product of the ASG conversations, held in July 2020. It is evidence of the University of Leicester's exceptionally collegiate and inclusive research environment, and the extraordinarily creative, confident thinkers who operate across our Colleges. It also speaks to the three values that underpin the pandemics thematic programme, and LIAS's mission within Leicester more widely: interdisciplinarity, inclusivity, and integrity.

As we navigate our way through the pandemic as a university - and indeed as a city - the ASG has shown that by providing structures and processes by which we can work together, we can achieve more collectively than we can individually. I am excited to see what the pandemics programme will deliver in the coming months. We are, in this moment, Citizens of Change. 
Why interdisciplinary approaches are essential in addressing pandemics and other global challenges: reflections from ASG Critical Friend, Dr Laura

\section{Meagher}

'Interdisciplinarity' is a word so often bandied about that at times it seems fated to lose its value. Yet, the pressing issue of today's global COVID-19 pandemic spotlights pandemics as a wicked problem that can only be addressed effectively through interdisciplinary approaches. Indeed, growing recognition of various critical global challenges underscores the increasing need for interdisciplinary approaches. This movement suits a pressing issue such as pandemics, which traverses geographical, national, social and individual barriers. As a way of thinking and working, interdisciplinarity inherently incorporates the hearing and valuing of diverse perspectives. A challenge jointly imposed across such a range requires using a research approach that does not shy away from such differences but rather helps coherent understanding to emerge from among them. This has both intellectual and operational implications.

On an intellectual level, for example in the framing of research problems, interdisciplinarity means embracing uncertainty in pursuit of clarity. This leads to accepting, becoming comfortable with or indeed enjoying work that takes place at intersections of broad themes and cross-cutting issues ... and, in so doing, finding a focus where a team's effort can add unique insight to a complex challenge. On an operational level, in framing and then conducting research efforts, interdisciplinarity means achieving synergy through collaboration shaped by mutual respect and a commitment to work through obstacles arising from different discipline-based worldviews.

When I facilitated the workshop in which the Pandemic Advanced Study Group identified priority themes, I was struck by manifestations of interdisciplinarity on both levels. Intellectually, members of the group shared ideas across their very different research fields and dug together into pandemics as a complex, multi-dimensional concept, doing the hard spadework of unearthing three valuable themes, each running across multiple facets and issues. Operationally, they brought to the task the intangible but vital resource of 'good will'. Their willingness to respect each other and each other's expertise allowed them to work effectively together, despite the complexity of the topic and the relative novelty of the virtual workshop format. As a member of the LIAS Advisory Group, I was especially pleased to see this, as it speaks to the development of a healthy culture of interdisciplinarity across the University of Leicester that will surely serve the university in good stead as it tackles pandemics and other global challenges into the future.

\section{Themes}

Three priority themes emerged from discussions held by the Pandemic Advanced Study Group (July 2020). These are broad topics, where i) interdisciplinary research will add significant value to our understanding of pandemics and their impacts, and ii) UoL already has a critical mass of worldleading scholarship. 


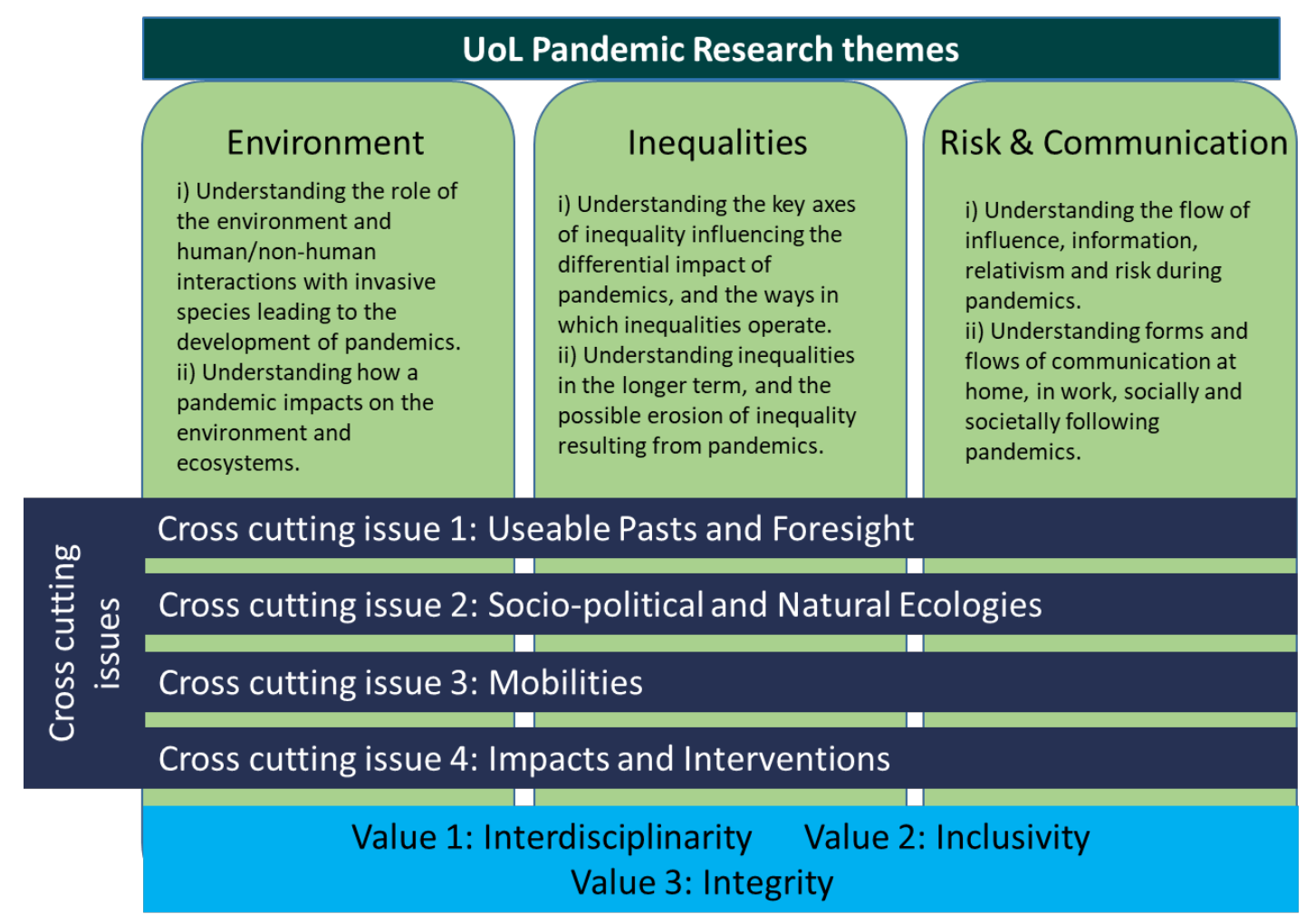

Figure 1: UoL Pandemics themes, issues and values

We expand on each of the three themes below, and conclude with a brief discussion of the four cross-cutting issues that have relevance to all of the themes.

Environment

Purpose of this research theme

This research theme invites understanding of (i) the role of the environment and human/ nonhuman interactions with invasive species leading to the development of pandemics and (ii) how a pandemic impacts on the environment and ecosystems.

COVID-19 is an invasive species that is causing widespread health, economic and potentially ecological harm. Environmental changes related to anthropogenic activities may be altering human susceptibility to infections as well as our likelihood of coming into contact with them. For example, the emergence of zoonotic infectious diseases can be correlated to a variety of factors, including habitat encroachment, fragmentation and alteration and direct human-wildlife interactions (Johnson et al., 2020).

Travelling humans have been involved in translocating invasive species over millennia, significantly altering ecologies. A key question is whether we can use the lessons learned from recent and historical pandemics to better understand and to manage our impact on ecologies, human and nonhuman, in the context of the current pandemic? Can we learn more about SARS-CoV-2 from the perspective and expertise of investigators who study species invasions over different timescales? What can be learned from previous pandemics, including those in non-human species?

A further question is around communication and how interconnectivity facilitates spread e.g. via air travel, and how disrupting interconnectivity also impacts on spread (for example PPE unable to reach low and middle-income countries). Could pandemics be seen as an inevitable form of control exerted by ecosystems to self-limit population growth (and overuse of the Earth's resources) with 
parallels with interchange of ecologies in other species in historical records, and what are the implications for this perspective on the way we view those suffering from the impacts of pandemics? How can we best understand the invasive nature of viruses - within humans or microbiomes - that have the potential for profound effects in wider ecologies? Are there recognized ecological circumstances that favour the emergence of invasive species? Can these be related to stabilising of ecological systems? How does this change our attitude to conservation practice and thinking about our relationship to non-humans?

A key question is considering the dynamics of usage of, and impacts on, natural resources - what are the long-term implications for human and non-human ecologies? For example, the COVID-19 pandemic is having a profound impact on the use of natural resources and also human impacts on the environment, for example, transport, plastics use and disposal, agriculture. Transport dropped by $>90 \%$ for some sectors leading to large - but transient - drops in air pollution. There is an opportunity to rebuild 'green' but this may be offset by subsidies to existing industries e.g. airlines and perceptions of risks from public transport use and the major demands on natural resources to service green energies (e.g. metals for wind turbines and solar energy) (Herrington, et al. 2019).

Another example is plastics, where there has been a dramatic increase in production for PPE and single use items to cut infection risk, but already there are reports of increased contamination of waterways from plastic waste, potentially related to behavioural issues - usage of PPE in sectors not trained in its use and responsible disposal. In data from China, during the pandemic medical waste, including a significant plastic proportion, increased by $370 \%$ in the Hubei Province although other waste (e.g. municipal solid waste) was reduced by around a third in large and medium cities (Klemes, et al. 2020).

Pandemics may reinforce and exacerbate geographical, socio-economic and minority ethnic inequalities. The current pandemic has put social inequalities into sharp focus, in terms of infection rate and severity but also highlighted issues around linked environmental inequalities such as access to green spaces and exposure to environmental degradation (e.g. plastic waste). Changes in behaviour with respect to the environment also need to be considered as they may be worse rather than better during and after the pandemic.

\section{University of Leicester distinctiveness}

The University is home to a number of groups and thematic strengths that speak to our unique ability to address this theme. The Anthropocene Research Group researches global challenges and exploring the complex ways in which socio-economic ecosystems impact the Earth System, linked to the Plastics research group which explores transport mechanisms, dispersal, fragmentation, deposition, degradation and burial globally and locally. Our Centre for Sustainable Resource Extraction has expertise in ore mineralogy, geochemistry and mineral exploration to targeting of new deposits for the required elements and develop tailored processes for their efficient and environmentally-sustainable extraction to service renewal energy infrastructure and the green economy, while UoL leads air quality research globally (including GIS, chemical transport modelling, satellite technology and Earth Observation, and population exposure assessment). Together, our NIHR Leicester Biomedical Research Centre, Centres for Environmental Health and Sustainability, Microbial and Infectious Diseases, and BME Health, and the Department of Respiratory Sciences and Institute of Lung Health explore the human health impacts of environmental exposures and how disease affects people from a myriad of ethnic backgrounds, including in relation to the respiratory microbiome. 
Inequalities

Purpose of this research theme

COVID-19 has amplified, accentuated, and arguably accelerated inequalities globally. This theme invites the academy to interrogate the complex landscape we will need to navigate over the coming years.

What are the key axes of inequality influencing the differential impact of the COVID-19 pandemic? In addition to normal 'fault lines' relating to ethnicity, migration status, social class, gender, sexuality, age, disability, how might other logics of social distribution have acquired significance, and new intersectional fault lines been exposed? Possible examples include child and elder care; access to green spaces, living alone, material conditions relating to housing, being able to work from home, job security, health risk inequalities (such as shielding).

In what ways might such inequalities operate: for example as enduring sources of disadvantage linked to respiratory morbidities; as differences shaping experiences of and responses to pandemic; as sources of marginalisation from the cultural imagery of public health messaging?

How might we understand such inequalities in the longer term, both in the UK but also in the Majority World ${ }^{1}$ ? For instance what are the continuities and discontinuities in inequalities? What is the role of existing inequalities 'going in', and the new inequalities 'coming out' of the pandemic? How, for instance, are certain inequalities amplified, compounded, and supplemented? What are the new 'fault lines' being produced by the pandemic both locally, nationally and globally? With relevance from Leicester to UK and global inequalities, Leicester is a global socio-cultural nexus that can act as a paradigm of broader cultural and geo-political divisions: from different local responses, different governmental responses, geo-political fault lines, different levels of coordination in testing, tracing, locking down, responsiveness, etc. What insights and new questions might we derive from adopting a much longer-term perspective? For example, what lessons can be drawn from experiences of, and economic and social responses to, medieval plagues or the Spanish Flu pandemic in the early 20th century? How, through adopting the perspective of geological time spans, might we understand the significance of COVID-19 and other past and possible future epidemics and pandemics in terms of, for instance, their increasing recurrence, their adaptation, and rapidity of spread? How might such timeframes, timespans, help us to understand inequalities within, between and across generations?

The inequalities theme also calls our attention to the temporalities bound up with inequalities; enduring axes of health inequalities and their longer-term genesis; rapid development of new divisions, new inequalities that both link to, compound, and add to enduring fault lines; possible future inequalities: trajectories of inequalities extrapolating from the present, globally and locally.

Finally, the inequalities theme uncovers the possible erosion of inequalities: how might 2020 involve a turning point in terms of lessons learned -- e.g. organisation of white collar workplaces; new ways of teaching and learning; sustainability of current practices relating to consumption (for example fast fashion and the practices of big brand retailers); problems of not enforcing health and safety and minimum wage legislation on the back of a 'deregulation' push;, human-animal relations and the increasing human encroachment into non-human habitats -- all of which have been thrown into sharp relief by the COVID-19 pandemic. By engaging across disciplines with the ways in which the pandemic has spread, we are presented with the opportunity to redefine what we mean by 'inequality' in a larger sense - beyond wealth, poverty, race, ethnicity, ability/disability.

\footnotetext{
${ }^{1}$ The terms 'developing countries', 'Global South', 'Third World', and 'low and middle income countries' are contested. In contrast and aligned with contemporary development research, we use the term 'Majority World' to describe the countries where most of the global population lives, but where least financial resource is located.
} 
The existing clinical expertise within the College of Life Sciences and, especially, the Centre for BME Health, as well as the need for an urgent response to the unique circumstances of Leicester's second COVID spike, means that the University is already at the cutting edge of investigating ethnic inequalities (and differential outcomes related to migration status) in relation to COVID-19. Notably, UoL leads the UK-REACH; the largest study to focus on ethnicity and COVID-19 in healthcare workers. The LIAS cross-College Migration, Mobility and Citizenship research network provides an experienced network with the capacity to address these issues. This work is already interdisciplinary, involving clinicians, biomedical scientists, computer, and social scientists. We now have the potential to draw on wider interdisciplinary expertise to think more expansively, across history and other pandemics, about the ways in which social inequalities of various kinds have shaped the impact of pandemics on different populations, but also how pandemics expose overlooked inequalities and make them urgent political issues. For example, the University's GCRF (Research England) allocation is now funding an exceptionally wide range of research on COVID-19, from the impact on Roma communities in Eastern Europe to developing an application for use in prisons in Guyana, whilst LIAS COVID-19 Urgency Grants have already brought together interdisciplinary and international teams to develop analyses of the experience of trans prisoners during lockdown, and the ways in which sexuality and gender have become known and contested during (and in relation to) the COVID-19 pandemic.

\section{Risk and communication}

\section{Purpose of this research area}

'Communications' offers a platform to investigate profound impacts of the construction and response to health messaging within both local and international contexts. The pandemic has highlighted emergent themes and challenges of communication on multiple levels; within families, within (new) workplaces, within healthcare and from government to the public. The impacts of these changes, and in particular the impacts on varied population groups, is not well understood.

Understanding communication within the whole population, both at home and at work, will be vital to the organisation of a post-COVID-19 society. In particular, the role of public health communication in pluralistic societies amplifies differential challenges surrounding population groups with regard to risk perception and risk management. When considering multi-ethnic public health communications and the dynamics of these perceptions, themes of inclusivity, inequality/alienation, cultural relevance and relativism offer critical focus points against and within dominant societal narratives. Resistance, socio-technical cognition and adherence are important factors when considering appropriate ways to manage the crisis. Channels of influence and information flow play an important role, with mis-information and misinterpretation having significant impacts on the outcomes of health messaging (for example, visible factors include lack of or poor peer review of scientific research as a result of rapidity of publication, and relativism / misinterpretation). Similarly, the tensions between communication-mobility freedoms and communication-mobility constraints, related impacts on all forms of health, and novel uses of existing platforms/creation of new platforms for communication. Questions surrounding access to resources within vulnerable groups affect cultural imagery (such as evoking Dunkirk imagery). Lastly, the temporal challenges of the pandemic highlight challenges in research communication, participation in research (e.g. pressure on survivors to take part in research) and the persistence of research engagement in the context of post-viral fatigue; these are all considerable factors in shaping and perceiving the effects of the pandemic, and messaging surrounding the pandemic.

Communication and information needs and responses vary across societies. For example: the same public health message may be perceived in very different ways by different groups, or there may be 
very different perceptions of a communication system (such as a GP's video consultation). Language and accessibility impact cognitive understanding and subsequent adherence to messaging guidelines. When we are tasked with measuring the effectiveness of health messaging, these considerations play a vital role. Furthermore, care and effort needs to be put in applying interdisciplinary design principles and approaches (e.g. User-centred Design (UCD) and Participatory Design (PD)) in designing and developing novel democratised communication platforms and interfaces that both address the emerging risks but also remain close to people's needs, requirements and experiences.

Universal wearing of PPE has led to overnight change in the way in which communication with patients, relatives and between professionals takes place. These changes have been made as a 'best guess' in a pressured situation - there has been no research on impact, perceptions or effectiveness of these changes. Some specific groups have obviously been impacted (for example deaf patients/ staff who are no longer able to lip read due to mask wearing), but there are many more subtle changes which have yet to be assessed. Access to healthcare and safe practice emerge as a human rights issue, and demonstrates disparities across societal groups.

Within the context of crises, perceptions of risk change over time manifesting a complex information landscape including notions of different health definitions and different levels of resistance (e.g. when asked to adhere to crisis management policies and actions). Identifying and managing these is critical in defining appropriate communication channels to mediate, address and support the communication of risks, at the right time, in the right way to everyone.

Social and work communication now has to take place in the context of isolation. Previously 'open' communities, such as nursing and residential homes, have suddenly become 'closed'. Social communication has radically changed due to externally applied rules, with individual choice about risk of communication methods being overtaken by the wider good of society. These changes are likely to have differential effects in different population groups, and offer a lens by which we can evaluate power and empowerment across subculture publics.

\section{University of Leicester Distinctiveness}

There are likely to be many research groups worldwide looking at the overall trends in communication ('Zoom studies'). However Leicester has the expertise to focus down on population subgroups. There is already a strong local focus on ethnicity research (for example the Biomedical Research Centre) with strong links into different communities. There is significant history expertise to bring in the learning from past pandemics. Business School expertise in workplace communication, together with strong clinical links through the College of Life Sciences that enable access to partner healthcare organisations and staff, will make it possible to investigate the social impact of messaging in multicultural environments. School of Informatics expertise in UCD and PD puts an emphasis on applying co-design methods to encourage and incorporate different stakeholders' needs (i.e. service providers, the public and local authorities) from different sociotechnical and ecological backgrounds in designing and developing communication technologies for decision-making in crises contexts. The prolonged local lockdown in Leicester gives us a nationally unique population to study, in particular for the temporal aspects and impacts of local lockdown in population groups. It is likely that local lockdown will be a future feature of a world with 'endemic COVID-19', so Leicester is well placed to research the effects to inform future communications policy.

\section{Cross-cutting issues}

Four cross-cutting issues emerged across the three ASG thematic discussion areas. 


\section{Usable pasts and foresight}

How might the past matter in the world today? This is the question that animates what some scholars have called the search for the usable past. Acknowledging the contested nature of the term, it is nonetheless indicative of a desire to connect history to present-day concerns, and to locate structures, patterns and experiences that can be brought to bear on solving contemporary issues and problems. Its relevance is potentially wide ranging and far reaching. Projects might include modelling the impact of Covid-19 from the vantage point of the Spanish Flu in the early $20^{\text {th }}$ century, examining the economic and social impact and recovery from plague, and assessing past attitudes to health and risk. Projects could take account of historical perspectives and deep time, including Earth observation and geological records, and archaeology. Alternatively, projects could seek to understand patterns and trends of communications across cities/nations, sociotechnical, cognitive, and behavioural contexts.

Closely related to our understandings of the past are the ways that we think about and plan for the future. In a research context, foresight enables us to: i) model, and think critically about, the longer term implications of the past and present; ii) build sets of competencies across groups of people from different disciplines and sectors; iii) act and shape the future, for example by supporting evidence-informed public policy or civic participation. Foresight activities usually consider a horizon at least ten years in the future, or even multiple alternative pathways of development (rather than only the most likely or maintenance of the status quo).

\section{Socio-political and natural ecologies}

This issue foregrounds the enormous variation of social and political contexts in which the COVID-19 pandemic has found footholds, both locally and globally. It acknowledges that pandemics cannot happen in a vacuum, but that families, communities, publics, the media, professionals, local and national authorities effect change to the health and wellbeing of individuals. It also provides a space in which we might explore the interplay between the biological, social, cultural, and environmental factors that contribute to pandemics, their evolution, behaviours, and impact. Finally, it explores the notion of 'ecological black box' that may manifest in crises situations whereby we need to unpack it to identify emerging needs, requirements, opportunities and constraints to consider when we design and build novel risk management technologies.

Projects could explore predispositions to pandemics, inequalities that occur or accelerate as a result of pandemics, and dominant structures, communal narratives, health messaging and social attitudes to death and disease. Drawing across the life and natural sciences, projects could understand invasions of microbial communities and ecosystems impacting health, or the links between ongoing conservation challenges such as habitat loss and fragmentation, wildlife trade and ways in which these are reshaping human/non-human contacts across different geographies and ecologies. This theme also seeks to set the biodiversity of the human microbiome in an ecological context.

\section{Mobilities}

The mobility cross-cutting issue invites exploration of how people, ideas, goals, systems, and viruses themselves change and evolve in pandemic circumstances. We consider mobility in the broadest sense of the term and on multiple levels, including the movement of people and infection through landscapes, time, and tangible/intangible structures, and social mobility. We consider the role technologies play or might play in facilitating dynamic mobility-enabled ecosystems that are supportive, accessible, and inclusive, and which are able to adapt and model fluidity in the environment. Mapping the provenance of such crises into tangible, usable and pleasant mobility 
infrastructures is paramount to safeguard and - potentially - enhance people's wellbeing during challenging times.

Projects could explore the ways globalisation affects the 'war on correct policy' and messaging between nations in a pandemic, interrogating differential economic stabilities and the ways they change and shape the messaging focus. They could also potentially expose which groups experience inclusion or alienation, particularly in the context of migration, mobility, and citizenship.

\section{Impacts and interdisciplinary interventions}

The COVID-19 pandemic has made life for some people, in some contexts, close to unbearable. As with previous pandemic, it has become clear that our understanding of the ways in which pandemics impact on different groups is still in its infancy. This cross-cutting issue focuses on understanding who is most affected by pandemics, why and where, and how to best mitigate impacts on the most seldom-heard groups.

Projects could explore the impacts and interventions needed to transform the course of, and recovery from pandemics, and the differential impacts and needs of societal groups.

\section{Conclusion}

This Working Paper has shared the reflections of the UoL Pandemics Advanced Study Group, who worked together during the highly unusual and challenging context of July 2020 . The framework we propose for future work potentiates interwoven, impactful, and nuanced understandings of the complexities of pandemics. Through the evolution of new knowledge with pragmatic implications, the city of Leicester, its cultural richness and the particular strengths of the University of Leicester, we can offer unique contributions to the understanding of pandemics now, and into the future.

\section{References}

Herrington, R., Boyce, A., Lusty, P., Murton, C., Naden, J., Roberts, S., Smith, D., Wall, F., (2019, 5 June) Letter to the Committee for Climate Change. https://www.nhm.ac.uk/press-office/pressreleases/leading-scientists-set-out-resource-challenge-of-meeting-net-zer.html

Johnson, C. K., P. L. Hitchens, P. S. Pandit, J. Rushmore, T. S. Evans, C. C. W. Young and M. M. Doyle (2020) Global shifts in mammalian population trends reveal key predictors of virus spillover risk. Proceedings of the Royal Society B: Biological Sciences 287(1924): 20192736.

Klemeš, J. J., Fan, Y. V., Tan, R. R., \& Jiang, P. (2020). Minimising the present and future plastic waste, energy and environmental footprints related to COVID-19. Renewable and Sustainable Energy Reviews, 127, 109883. 